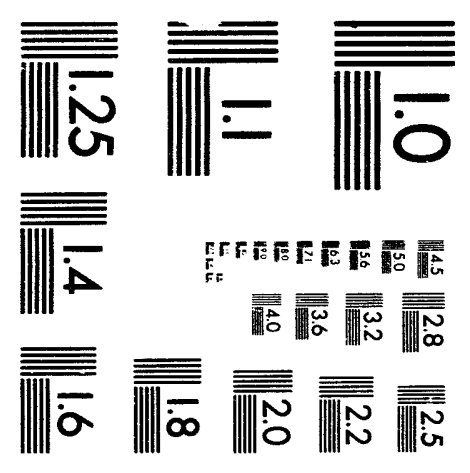



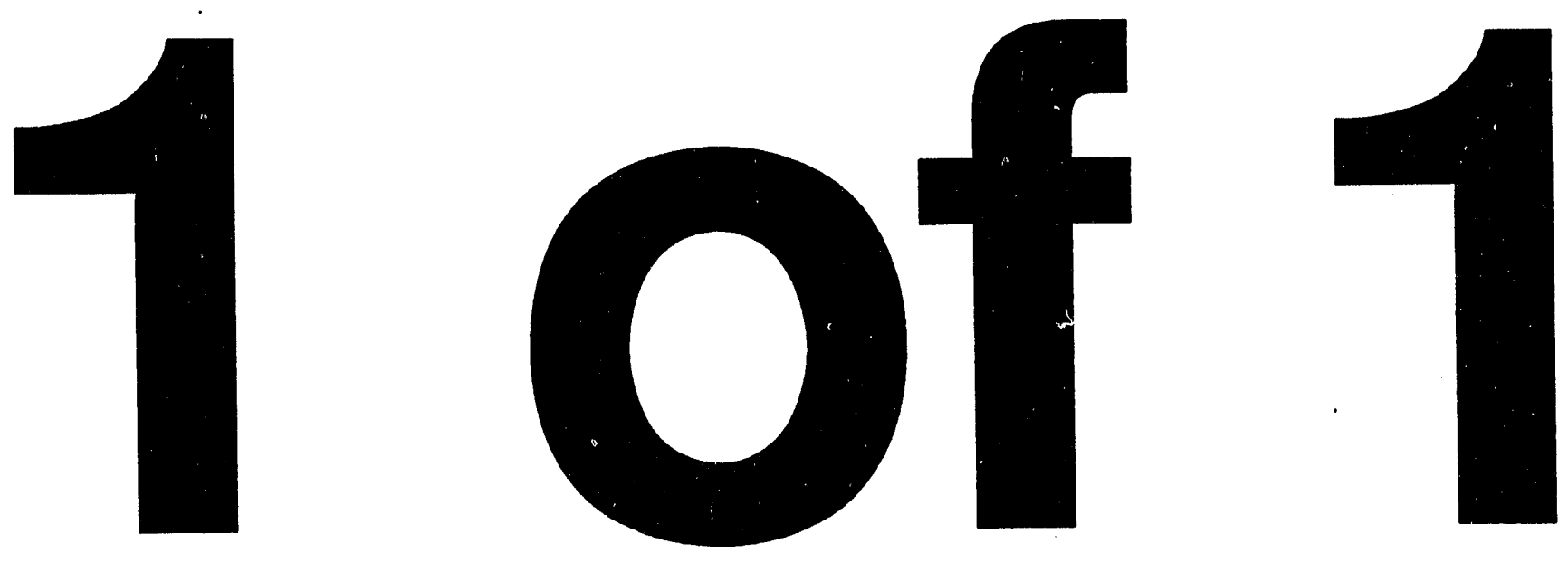


\title{
FUEL ION ROTATION MEASUREMENT AND ITS IMPLICATIONS ON H-MODE THEORIES
}

\author{
by \\ J. KIM, K.H. BURRELL, P. GOHIL, \\ R.J. GROEBNER, F.L. HINTON, Y.B. KIM, \\ W. MANDL, ${ }^{*}$ R. SERAYDARIAN, and M.R. WADE ${ }^{\dagger}$
}

This is a preprint of a paper to be presented at the $4^{\text {th }}$ International $\mathrm{H}$-Mode Workshop, November 15-17, 1993, Ibaraki-ken, Japan, and to be printed in the Proceedings.

Work supported by

U.S. Department of Energy

Contract No. DE-AC03-89ER51114

* Permanent address: Max Planck Institute für Plasma Physics, Garching, Germany.

$\dagger$ Permanent address: Oak Ridge National Laboratory, Oak Ridge, Tennessee.

\section{GENERAL ATOMICS PROJECT 3466 OCTOBER 1993}

\section{GENERAL ATOMICS




\title{
Fuel ion rotation measurement and its implications on $\mathrm{H}$-mode theories*
}

\author{
J. Kim, K.H. Burrell, P. Gohil, R.J. Groebner, F.L. Hinton, Y.B. Kim, \\ W. MANDL ${ }^{\dagger}$ R. SERAYDARIAN, AND M.R. WADE ${ }^{\ddagger}$ \\ General Atomics, P.O. Box 85608, San Diego, California 92186-9784, U.S.A.
}

(Received

\begin{abstract}
Poloidal and toroidal rotation of the fuel ions $\left(\mathrm{He}^{2+}\right)$ and the impurity ions $\left(\mathrm{C}^{6+}\right.$ and $\left.\mathrm{B}^{5+}\right)$ in $\mathrm{H}$-mode helium plasmas have been investigated in the DIII-D tokamak by means of charge exchange recombination spectroscopy, resulting in the discovery that the fuel ion poloidal rotation is in the ion diamagnetic drift direction while the impurity ion rotation is in the electron diamagnetic drift direction. The radial electric field obtained from radial force balance analysis of the measured pressure gradients and rotation velocities is shown to be the same regardless of which ion species is used and therefore is a more fundamental parameter than the rotation flows in studying $\mathrm{H}$-mode phenomena. It is shown that the three contributions to the radial electric field (diamagnetic, poloidal rotation, and toroidal rotation terms) are comparable and consequently the poloidal flow does not solely represent the $E \times B$ flow. In the high-shear edge region, the density scale length is comparable to the ion poloidal gyroradius, and thus neoclassical theory is not valid there. In view of this new discovery that the fuel and impurity ions rotate in opposite sense, $\mathrm{L}-\mathrm{H}$ transition theories based on the poloidal rotation may require improvement.
\end{abstract}

\section{Introduction}

Since its original discovery in ASDEX (Wagner et al. 1982, 1985), the H-mode has proven to be one of the most robust and ubiquitous modes of improved confinement in toroidal magnetic fusion devices. The physics of the $\mathrm{L}$-mode to $\mathrm{H}$-mode transition ( $\mathrm{L}-\mathrm{H}$ transition) has attracted a great deal of interest and effort from both the experimental and theoretical communities. The most widely accepted paradigm thus far is that a highly sheared radial electric field is formed in the plasma edge in $\mathrm{H}$-mode and the resulting sheared $E \times B$ flow can lead to better confinement through decorrelation of the fluctuations, decreased radial correlation lengths and reduced turbulent transport (Burrell et al. 1992, Groebner 1993, Biglari et al. 1990, Shaing and Crume 1989). The experimental observations that the edge impurity ion poloidal rotation (Groebner et al. 1991, Doyle et al. 1991, Ida et al. 1990) and the edge radial electric field (Groebner et al. 1991, Doyle et al. 1991, Ida et al. 1990, Taylor et al. 1989, Van Nieuwenhove et al. 1991) change dramatically at the L-H transition have led to several theories which consider how the radial electric field or the (fuel) ion poloidal rotation changes across the L-H transition. Among these are theories based on bifurcation of the radial electric field (Shaing and Crume 1989, Itoh and Itoh 1990), Stringer spin-up (Hassam et al. 1990, Stringer 1969), turbulent Reynolds stress (Diamond and Kim 1991), temperature gradient-induced poloidal rotation (Hinton 1991), particle and energy confinement bifurcation (Hinton and Staebler 1993).

* Work supported by the U.S. Department of Energy under Contract DE-AC03-89ER51114.

$\dagger$ IPP, Garching, Germany.

$\ddagger$ Oak Ridge National Laboratory, Oak Ridge, Tennessee. 
One other complication in the history of L-H transition studies was due to the fact that only the impurity ion rotation measurements had been available previously because of difficulty in measuring the fuel ion (deuterium or hydrogen) rotation. Most L-H transition theories set out to explain the sudden increase of poloidal rotation in the electron diamagnetic direction, assuming that the main ion rotation is identical to the impurity ion rotation. However, recent spectroscopic measurements in helium $\mathrm{H}$-mode plasma in the DIII-D tokamak have shown that the fuel ions rotate in the ion diamagnetic direction at a speed as large as about $30 \%$ of the ion diamagnetic speed, which is opposite to the impurity rotation direction (Kim et al. 1993).

Actually the neoclassical theory predicts the fuel ion poloidal rotation to be in the ion diamagnetic direction in the banana regime (Hinton and Hazeltine 1976), and a recent derivation of rotation speeds for a system of two ion species shows that the sign of the fuel and impurity poloidal rotation can be different depending on the collisionality (Kim et al. 1991). The neoclassical poloidal rotation prediction is fairly good for the impurity ions; but is much smaller than the fuel ion rotation (Kim et al. 1993). The measurement also showed ihat an appreciable difference in toroidal rotation between the fuel and the impurity ion exists in the edge region. An extended neoclassical theory of rotation with the orbit squeezing effect, which reduces the parallel flow, yields a prediction much closer to the measured ones (Hinton et al. 1993). In this theory, the fuel ion poloidal rotation depends not only on the temperature gradient, but also on the density gradient.

In this paper, we first summarize the result of fuel ion rotation measurements in the DIII-D tokamak where helium was employed as the main fuel ions. Charge exchange recombination (CER) spectra of helium lines (He II) represent the fuel ions; carbon lines (C VI) and boron lines $(\mathrm{B} V$ ) represent the impurity ions. The experimental results and the neoclassical poloidal rotation predictions are compared. The implications of these results on L-H transition theories and $\mathrm{H}$-mode phenomenology are then discussed.

\section{Experiment and analysis}

The CER system (Groebner et al. 1990, Gohil 1991) on the DIII-D tokamak allows us to monitor any select spectral line emitted from excited ions localized where the line of sight and the neutral beam cross. These spectral lines contain information on the Doppler broadening (temperature) and the Doppler shift (rotation speed). The spatial coverage ranges from $R=$ $1.77 \mathrm{~m}$ to $R=2.30 \mathrm{~m}$. Deuterium neutral beam injection (NBI) was chosen to provide CER for $\mathrm{He}^{2+}\left[\mathrm{He}^{2+}+\mathrm{D}^{\circ} \rightarrow\left(\mathrm{He}^{+}\right)^{*}+\mathrm{D}^{+}\right]$and to force the $\mathrm{L}-\mathrm{H}$ transition in helium plasmas. NBI was modulated to allow subtraction of the plasma emission (without beem) from the total emission (with beam) to yield pure CER spectra. Carbon and boron are the primary impurities in the DIII-D tokamak whose graphite inner wall was boronized. In addition, $\mathrm{D}^{+}$ions from the injected beams constitute an impurity in helium plasma, $\mathrm{D}^{+}$concentration being about $10 \%$ of the electrons around the time of $\mathrm{L}-\mathrm{H}$ transition and rising monotonically with time into NBI pulse. The unshifted wavelength locations needed for obtaining Doppler shifts were determined by the line locations of the spectra during the Ohmic phase of the shot. Fiducials obtained in this way were validated by using a pair of opposing tangential chords focussed at the same major radius point.

Some plasma parameters of interest are $B_{\phi}=-1 \mathrm{~T}$ to $-2 \mathrm{~T}, n_{\mathrm{e}}=1-4 \times 10^{19} \mathrm{~m}^{-3}, I_{\mathrm{p}}=$ $1 \mathrm{MA}$, and $P_{\mathrm{inj}}=4-6 \mathrm{MW}$. The collisionality parameter $\left(\nu_{\mathrm{ii}}^{*}\right)$ ranges from 0.1 to 0.3 in the edge region well after the $\mathrm{L}-\mathrm{H}$ transition. Photodiode signals show the onset of a dithering $\mathrm{L}-\mathrm{H}$ transition at around $t=2330 \mathrm{~ms}$, about $30 \mathrm{~ms}$ into the NBI pulse. In the $(r-\theta-\phi)$ coordinate where $\theta$ is the poloidal direction and $\phi$ is the toroidal direction, the sign convention of the present experiment and analysis is as follows: $B_{\phi}$ is negative, the plasma current $I_{\mathrm{p}}$ is in the positive $\phi$-direction, NBI is co-injection with respect to $I_{\mathrm{p}}$, the toroidal rotation is positive, 
$B_{\theta}$ is positive (i.e. downward at the outboard midplane), the ion diamagnetic flow is in the positive $\theta$-direction, and the electron diamagnetic flow is in the negative $\theta$-direction.

The standard neoclassical poloidal rotation of the fuel ions $\left(V_{\theta \mathrm{i}}\right)$ and the impurity ions $\left(V_{\theta \mathrm{I}}\right)$ for the three-species plasma are given in Kim et al. (1991). Input to the theoretical expressions are the measured quantities, such as, the density scale length $\left(L_{n i}\right)$, the temperature scale length, and local magnetic fields. Both the temperature profile and the density profile are obtained from CER spectroscopy. The collisionality parameter $\left(\nu^{*}\right)$ is calculated using a generalized definition (Kim et al. 1991) rather than the usual large aspect-ratio approximation. Local and flux-surface averaged magnetic fields used in the theoretical computation are obtained from MHD equilibrium analysis (Lao et al. 1985).

The radial electric field is derived from the equilibrium force balance equation. The same equation is also valid for the impurity ion (by changing the subscript from i to I).

$$
E_{r}=\frac{1}{n_{\mathrm{i}} Z_{\mathrm{i}} e}\left(\frac{d P_{\mathrm{i}}}{d r}\right)-V_{\theta \mathrm{i}} B_{\phi}+V_{\phi \mathrm{i}} B_{\theta}
$$

where $Z_{i} e$ is the charge of the ion. Since $P_{\mathrm{i}}$ decreases with $r$, the first term is negative.

\section{Result of measurement and calculation}

Measured poloidal rotation velocities of $\mathrm{He}^{2+}$ and $\mathrm{C}^{6+}$ in the edge region about $150 \mathrm{~ms}$ after the $\mathrm{L}-\mathrm{H}$ transition are shown in Fig. 1. The directions of the poloidal rotations are in agreement with the neoclassical predictions. $V_{\theta \mathrm{i}}$ is in ion diamagnetic direction, while $V_{\theta \mathrm{I}}$ is in the usual electron diamagnetic direction. $V_{\theta \mathrm{i}}$ is appreciably faster than $V_{\theta \mathrm{I}}$. Neoclassical predictions for $V_{\theta \mathrm{i}}$ and $V_{\theta \mathrm{I}}$ are also plotted in Fig. 1. It is noted, however, that $L_{\mathrm{ni}} \sim 1 \mathrm{~cm}$ and $\rho_{\theta \mathrm{i}} \sim 2 \mathrm{~cm}$ in the edge region a few $\mathrm{cm}$ within the separatrix, and thus the neoclassical theory, which is based on the assumption of $L \gg \rho_{\theta i}$, may not be valid there.

The measured toroidal rotations, $V_{\phi \mathrm{i}}$ and $V_{\phi \mathrm{I}}$, although not shown in this paper, also exhibit different behavior in the edge: $V_{\phi \mathrm{i}}$ remains relatively large even around the separatrix whereas $V_{\phi \mathrm{I}}$ decreases more rapidly towards the separatrix (Kim et al. 1993).

Because $E_{r}$ is the property of the overall plasma common to any species, one should expect an identical result from all the ion species. This very fact, therefore, serves also as a consistency check on the experimental results. Shown in Fig. 2 are the $E_{r}$ profiles inferred from $\mathrm{He}^{2+}, \mathrm{C}^{6+}$, and $\mathrm{B}^{5+}$, respectively. Considering the different rotation characteristics of the fuel ions and the impurity ions, the agreement among the three independent $E_{r}$ profiles is quite remarkable. In Fig. 3, the contribution from each of the three terms of the $E_{r}$ equation is compared for the case of the fuel ion. A negative well shape of $E_{r}$ in the edge region is maintained by the dominant pressure gradient term against the

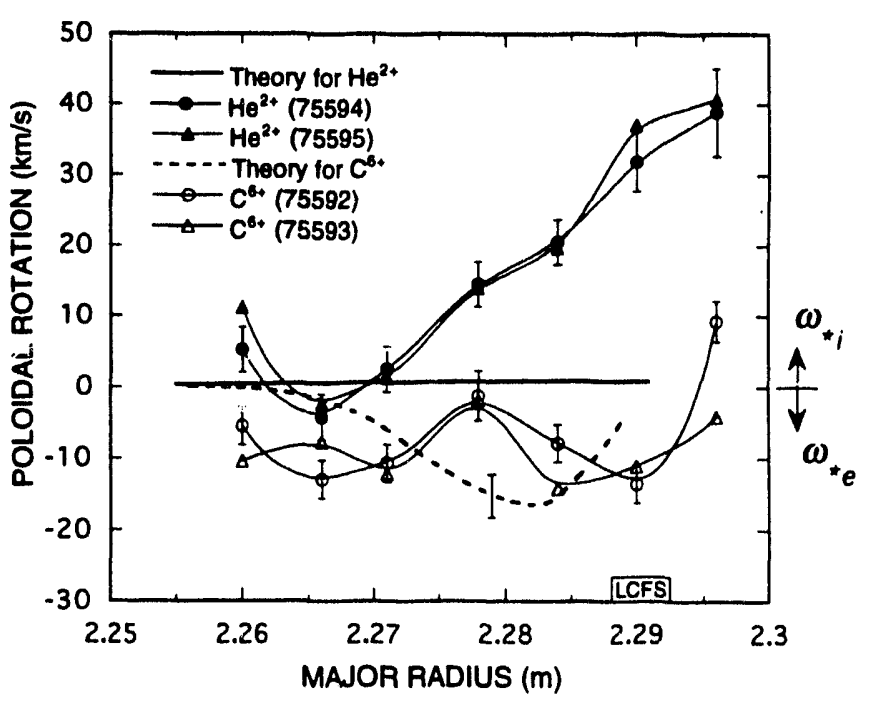

Figure 1. Measured and calculated poloidal rotation of the fuel ions $\left(\mathrm{He}^{2+}\right)$ and the impurity ions $\left(\mathrm{C}^{6+}\right)$ in the edge region about $150 \mathrm{~ms}$ after the L-H transition. The last closed flux surface (LCFS) varies somewhat from shot to shot. 
positive contribution from $-V_{\theta \mathrm{i}} \mathrm{B}_{\phi}$ and $V_{\phi i} \mathrm{~B}_{\phi}$ terms. For the impurity ion, although not shown here, the pressure gradient term and $-V_{\theta \mathrm{I}} \mathrm{B}_{\phi}$ term are both negative against the positive contribution from $V_{\phi \mathrm{I}} \mathrm{B}_{\theta}$ term. It is noted that the impurity pressure gradient is smaller than the fuel ion and its effect is further reduced by the factor, $Z_{\mathrm{i}} / Z_{\mathrm{l}}$.

The evolution of $V_{\theta i}$ and $E_{r}$ across the L-H transition is shown in Fig. 4. $V_{\theta i}$ is seen to jump in the ion diamagnetic direction as early as $3 \mathrm{~ms}$ after the $\mathrm{L}-\mathrm{H}$ transition. Before the transition the radial electric field is nearly flat across the edge region, and then a negative well shape in the shear region is formed immediately after the transition even though the poloidal rotation jump in the ion diamagnetic direction contributes toward the positive electric field. The electric field continues to evolve slowly with time into the $\mathrm{H}$-mode towards a more negative well as the pressure gradient builds up more.

\section{Implications on $\mathbf{H}$-mode theories}

The result of the measurement and theoretical computations given above has revealed several new discoveries. These findings not only concern the rotation theories, but have some subtle implications on $\mathrm{H}$-mode theories and phenomenology as follows.

a. Several theories (Shaing and Crume 1989, Hassam et al. (1991), Hinton 1991) attempted to explain the connection between the L-H transition and a $V_{\theta i}$ jump in the electron diamagnetic direction. However, the measurement showed $V_{\theta i}$ to jump in the ion diamagnetic direction right after $(\sim 3 \mathrm{~ms})$ the $\mathrm{L}-\mathrm{H}$ transition. It is now apparent that $V_{\theta i}$ and $V_{\theta I}$ need to be distinguished.

b. The three contributions to the radial electric field (diamagnetic, poloidal rotation, and toroidal rotation terms) are rather comparable and consequently the poloidal flow does not solely represent the $E \times B$ flow. Some theories (Biglari et al. 1990, Diamond and Kim 1991 ) opted not to distinguish the $E \times B$ flow and the poloidal rotation.

c. The radial electric field was shown to be the same regardless of which ion species is used to deduce it. Therefore $E_{r}$ is a more fundamental parameter than the rotation flows 
themselves. As long as $E_{r}$ is used in the study of $\mathrm{H}$-mode, the impurity ion measurement are as useful as the fuel ion measurement which is usually much harder to obtain.

d. In the theory of Shaing, et al. (1989) the ion orbit loss is the primary driver for $\mathrm{L}-\mathrm{H}$ transition. Solving Eq. (6) of the above reference for the poloidal rotation, however, it can be seen that the ion orbit loss term forces $V_{\theta i}$ to be in the electron diamagnetic direction; we have not yet seen the fuel ion poloidal rotation in that direction right after L-H transition.

e. In the very edge region of the $\mathrm{H}$-mode plasma with a large highshear, the validity of the neoclassical theory becomes questionable since it requires $L \gg \rho_{\theta \mathrm{i}}$. Inclusion of the orbit squeezing effect Hinton et al. submitted to Phys. Rev. Lett.) increased the neoclassical prediction by an order of magnitude there.

f. Stringer spin-up theory predicts $V_{\theta i}$ in the electron diamagnetic direction (McCarthy et al. 1993) contrary to our observations.

\section{Conclusions}

Measurement of poloidal and toroidal rotation of the fuel ions and the impurity ions in $\mathrm{H}$-mode helium plasmas revealed that the fuel ion poloidal rotation is in the ion diamagnetic drift direction while the impurity ion rotation is in the electron diamagnetic drift direction in contrast to the general notion that they rotate the same. The inferred radial electric field is shown to be the same regardless of which ion species is used. The $E \times B$ flow is not primarily represented by the fuel ion poloidal flow, but depends more on the fuel ion pressure gradient. In the high-shear edge region, the density scale length is comparable to the ion poloidal gyroradius, and thus neoclassical theory may not be applicable there.
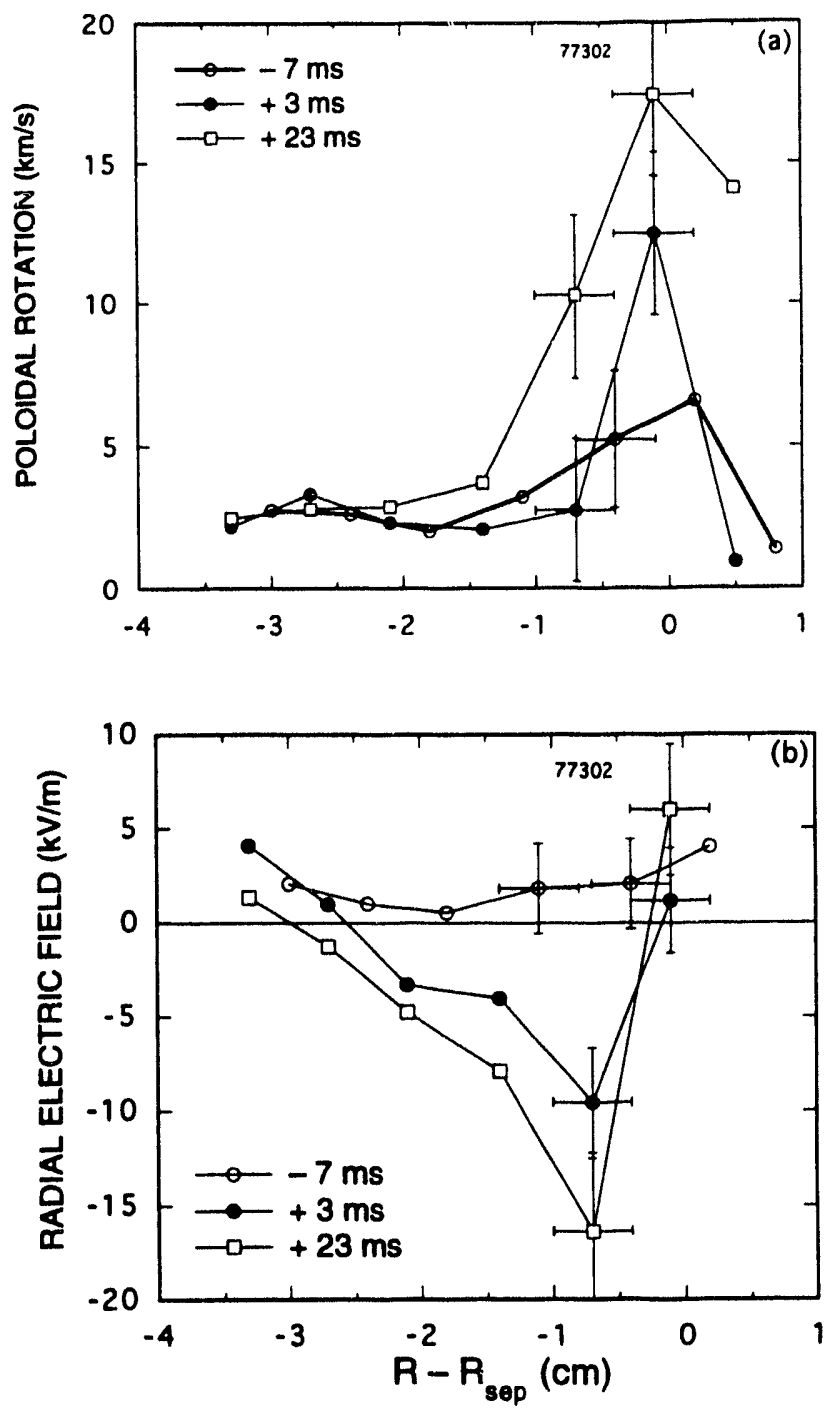

Figure 4. Fuel ion poloidal rotation and electric field before and after the L-H transition $(t=0)$ in the edge region. 


\section{References}

Biglari, H., Diamond, P.H., and Terry, P.W. (1990) Phys. Fluids B 2, 1.

Burrell, K.H., Carlstrom, T.N., Doyle, E.J., Finkenthal, D., Gohil, P., Groebner, R.J., Hillis, D.L., Kim, J., Matsumoto, H., MoYeR, R.A., Osborne, T.H., Rettig, C.L., Peebles, W.A., Rhodes, T.L., St. John, H., Stambaugh, R.D., Wade, M.R., and Watkins, J.G. (1992) Plasma Physics and Controlled Fusion 34, 1859.

Diamond, P.H., and KIM, Y.B., (1991) Phy s. Fluids B 3, 1626.

Doyle, E.J., Groebner, R.J., Burrell, K.H., Gohil, P., Lehecka, T., LuhmanN, Jr., N.C., Matsumoto, H., Osborne, T.H., Peebles, W.A., and Philipona, R. (1991) PhyS. Fluids B 3, 2300.

Gohil, P., Burrell, K.H., Groebner, R.J., Kim, J., Martin, W.C., MCKee, E.L., AND SERAYDARIAN, R.P. (1992) 14th Symp. on Fusion Engineering, Vol. II, P. 1189 (IEEE, NEW YORK).

Groebner, R.J., Burrell, K.H., Seraydarian, R.P. (1990) Phys. Rev. Lett. 64, 3015. Groebner, R.J., Burrell, K.H., Gohil, P., Seraydarian, R. (1990) Rev. Sci. Instrum. 61, 2920.

Groebner, R.J., Peebles, W.A., Burrell, K.H., Carlstrom, T.N., Gohil, P., SerayDarian, R.P., Doyle, E.J., Philipona, R., Matsumo'to, H., and Cluggish, C. (1991) Plasma Physics and Controlled Nuclear Fusion Research 1990, Vol. 1, P. 453 (IAEA, VIENNA).

Groebner, R.J. (1993) Phys. Fluid B 5, 2343.

Hassam, A.B., Antonsen, JR., T.M., Drake, J.F., ANd LiU, C.S. (1991) Phys. Rev. Lett. 66, 309.

Hinton, F.L., Hazeltine, R.D. (1976) Rev. of Mod. Phys. 48, 239.

Hinton, F.L. (1991) Phys. Fluids B 3, 696.

Hinton, F.L., Staebler, G.M., (1993) Phys. Fluids B 5, 1281.

Hinton, F.L., Kim, J., Kim, Y.-B., et al., SUBMitTed to Phys. Rev. Lett.

IdA, K., Hidekuma, S., Miura, Y., et al. (1990) Phys. Rev. Lett. 65, 1364.

ItoH, S.-I., AND ITOH, K., (1990) Phys. Soc. Japan 59, 3815.

Kim, Y.-B., Diamond, P.H., AND Groebner, R.J. (1991) Phys. Fluids B 3, 2050.

Kim, J., Eurrell, K.H., Gohil, P., Groebner, R.J., Kim, Y.B., St. John, H., SerayDARIAN, R.P., AND WADE, M.R. SUBMITTED TO Phys. Rev. Lett.

LaO, L.L., St. John, H., Stambaugh, R.D., et al. (1985) Nucl. Fusion 25, 1611.

MCCARThy, B.R., et al. (1993), Phys. Fluids B 5, 1188.

Shaing, K.C., And Crume, Jr., E.C.(1989) Phys. Rev. Lett. 63, 2368; Shaing, K.C.,

Crume, Jr., E.C., ANd Houlberg, W.A. (1990) Phys. Fluids B 2, 1492.

Stringer, T.E., (1969) Phys. Rev. Lett. 22, 770.

TAYlor, R.J., Brown, M.L., Fried, B.D., et al. (1989) Phys. Rev. Lett. 63, 2365.

Van Nieuwenhove, R., Van Oost, G., Weynants, R.R. etal. (1991) 18th Europ. Conf.

Contr. Fus. and Plasma Physics, Vol. 15C, p. 405.

Wagner, F., Becker, G., Behringer, K., et al., (1982) Phys. Rev. Lett. 49, 1408.

Wagner, F., Bartiromo, R., Becker, G., et al. (1985) Nucl. Fusion 25, 1490. 

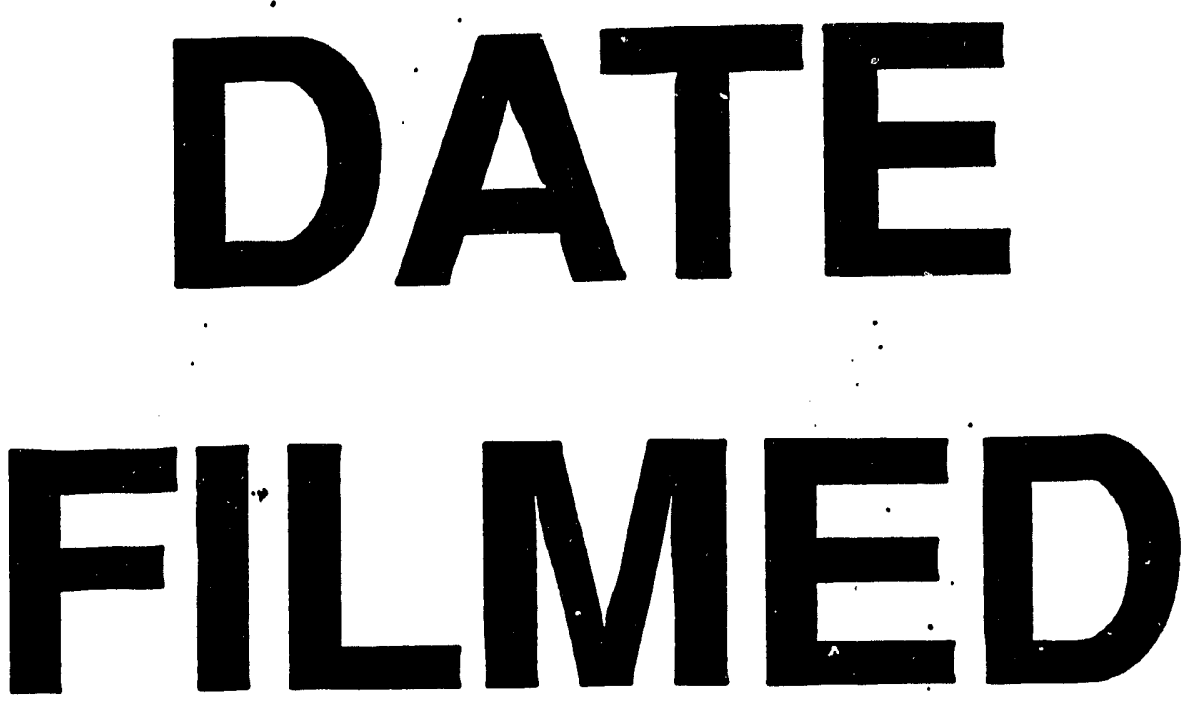

$2 / 4 / 94$
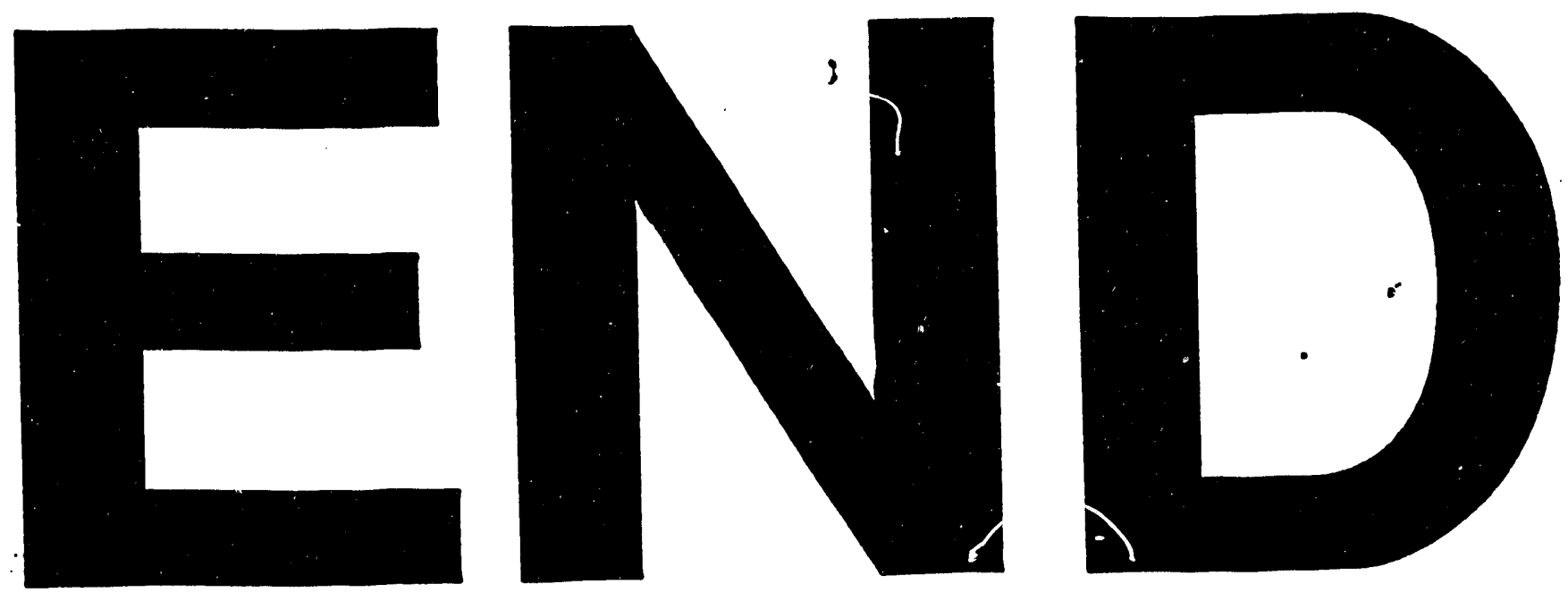
